International Mathematical Forum, 1, 2006, no. 38, 1871-1880

\title{
Notes on the Graded Prime Submodules
}

\author{
S. Ebrahimi Atani and F. Farzalipour \\ Department of Mathematics \\ University of Guilan \\ P.O. Box 1914 Rasht Iran
}

\begin{abstract}
Let $G$ be a monoid with identity $e$, and let $R$ be a $G$-graded commutative ring. Here we study the graded prime submodules of a graded $R$-module. While the bulk of this work is devoted to extending some results from prime submodules to graded prime submodules. A number of results concerning of these class of submodules are given.
\end{abstract}

Mathematics Subject Classification: 13A02, 16W50

Keywords: Graded rings, Graded prime submodules

\section{Introduction}

Several authors have extended the notions of prime and primary ideals to modules (see [1]-[9]). In this paper, we continue this investigations for graded prime and graded primary submodules of a graded module over a $G$-graded ring. Various properties of such modules are considered (see section 2).

Before we state some results let us introduce some notation and terminology. Let $G$ be an arbitrary monoid with identity $e$. A commutative ring $R$ with non-zero identity is $G$-graded if it has a direct sum decomposition (as an additive group) $R=\oplus_{g \in G} R_{g}$ such that $1 \in R_{e}$ and for all $g, h \in G, R_{g} R_{h} \subseteq R_{g h}$. If $R$ is $G$-graded, then an $R$-module $M$ is said to be $G$-graded if it has a direct sum decomposition $M=\oplus_{g \in G} M_{g}$ such that for all $g, h \in G, R_{g} M_{h} \subseteq M_{g h}$. An element of some $R_{g}$ or $M_{g}$ is said to be homogeneous element. A submodule of $N \subseteq M$, where $M$ is $G$-graded, is called $G$-graded if $N=\oplus_{g \in G}\left(N \cap M_{g}\right)$ or if , equivalently, $N$ is generated by homogeneous elements. Moreover, $M / N$ becomes a $G$-graded module with $g$-component $(M / N)_{g}=\left(M_{g}+N\right) / N$ for 
$g \in G$. Clearly, 0 is a graded submodule of $M$. Also, we write $h(R)=\cup_{g \in G} R_{g}$ and $h(M)=\cup_{g \in G} M_{g}$. A graded ideal $I$ of $R$ is said to be graded prime ideal if $I \neq R$; and whenever $a b \in I$, we have $a \in I$ or $b \in I$, where $a, b \in h(R)$. The graded radical of $I$, denoted by $\operatorname{Gr}(I)$, is the set of all $x \in R$ such that for each $g \in G$ there exists $n_{g}>0$ with $x_{g}^{n_{g}} \in I$. A graded ideal $I$ of $R$ is said to be graded primary ideal if $I \neq R$; and whenever $a, b \in h(R)$ with $a b \in I$, then $a \in I$ or $b \in G r(I)$. In this case, $G r(I)=P$ is a graded prime ideal of $R$, and we say that $I$ is a graded $P$-primary ideal of $R$ (see [8, Lemma 1.8]. The graded radical of a graded submodule $N$ of a graded module $M$ denoted $\operatorname{Gr}(N)$ and is defined to be intersection of all graded prime submodules of $M$ containing $N$. Clearly, if $N$ and $K$ are graded submodules of $M$ with $K \subseteq N$, then $\operatorname{Gr}(K) \subseteq G r(N)$.

\section{Graded prime submodules}

Our starting point is the following definition:

Definition 2.1 Let $R$ be a G-graded ring, $M$ a graded $R$-module, $N$ a graded $R$-submodule of $M$.

(i) We say that $M$ is a graded torsion-free $R$-module whenever $a \in h(R)$ and $m \in h(M)$ with am $=0$ implies that either $a=0$ or $m=0$.

(ii) $N$ is a graded prime submodule of $M$ if $N \neq M$; and whenever $a \in h(R)$ and $m \in h(M)$ with am $\in N$, then either $m \in N$ or $a \in\left(N:_{R} M\right)$.

(iii) $N$ is a graded primary submodule of $M$ if $N \neq M$; and whenever $a \in h(R)$ and $m \in h(M)$ with am $\in N$, then either $m \in N$ or $a^{k} \in\left(N:_{R} M\right)$ for some $k$.

(iv) $N$ is a graded maximal submodule of $M$ if $N \neq M$ and there is no graded submodule $K$ of $M$ such that $N \nsubseteq K \nsubseteq M$.

The following lemma is known, but we write it here for the sake of references.

Lemma 2.2 Let $R$ be a G-graded ring, $M$ a graded $R$-module and $N, K$ graded $R$-submodules of $M$. The following hold:

(i) If $I$ and $J$ are graded ideals of $R$, Then $(N: M), I J, I+J$ and $I \cap J$ are graded ideals of $R$.

(ii) If $r \in h(R)$ and $x \in h(M)$, then $R x, I N$ and $r N$ are graded submodule of $M$. 
Let $R$ be a $G$-graded ring. We say that $R$ is a graded integral domain whenever $a, b \in h(R)$ with $a b=0$ implies that either $a=0$ or $b=0$. A $G$-graded ring $R$ is said to be $G$-graded field if $0 \neq a \in h(R)$, then $a b=1$ for some $b \in h(R)$.

Lemma 2.3 Let $R$ be a non-trivial $G$-graded ring, $M$ a graded $R$-module and $P$ a graded ideal of $R$. The following hold:

(i) $R$ has at least one graded maximal ideal. In particular, if $I$ is a proper graded ideal of $R$, then there exists a graded maximal ideal $Q$ of $R$ with $I \subseteq Q$.

(ii) $P$ is a graded maximal ideal of $R$ if and only if $R / P$ is a graded field.

(iii) If $R$ is a graded field, then $M$ is a graded torsion-free.

(iv) $R$ is a graded field if and only if $R$ has exactly two graded ideals.

Proof. The proof is straightforward.

Proposition 2.4 Let $R$ be a $G$-graded ring, $M$ a graded $R$-module and $N$ a graded $R$-submodule of $M$. The following hold:

(i) If $(N: M)=P$ is a graded maximal ideal of $R$, then $N$ is a graded prime submodule of $M$.

(ii) If $Q$ is a graded maximal ideal of $R$ with $Q M \neq M$, then $Q M$ is graded prime.

Proof. (i) By Lemma 2.3, $M / N$ is a graded torsion-free over the graded field $R / P$, so $N$ is prime by [2, Theorem 2.11].

(ii) since $Q \subseteq(Q M: M)$, we must have $(Q M: M)=Q$. Now the assertion follows from (i).

Theorem 2.5 Let $R$ be a G-graded ring, $M$ a graded $R$-module and $N, N_{1}$, ..., $N_{k}$ graded $R$-submodules of $M$. If $N$ is graded prime with $\bigcap_{i=1}^{k} N_{i} \subseteq N$, then either $N_{i} \subseteq N$ or $\left(N_{i}: M\right) \subseteq(N: M)$ for some $i$.

Proof. Suppose not. Then there exists a homogeneous element $a \in N_{1}$ and for each $i \neq 1$, there are homogeneous elements $b_{i} \in\left(N_{i}: M\right)$ such that $b_{i} \notin(N: M)$ and $a \notin N$, so $b_{2} b_{3} \ldots b_{k} a \in \bigcap_{i=1}^{k} N_{i} \subseteq N$; hence $N$ graded prime gives $b_{2} b_{3} \ldots b_{k} \in(N: M)$ which is a contradiction, as required.

Let $M$ be a graded module over a $G$-graded ring $R$. Now consider the subset $T(M)$ of $M$ is defined by

$$
T(M)=\{m \in M: r m=0 \text { for some } 0 \neq r \in h(R)\}
$$


If $R$ is a graded integral domain, then $T(M)$ is a graded $R$-submodule of $M$ (see [2, Proposition 2.5]).

Proposition 2.6 If $R$ is a graded integral domain and $M$ a graded $R$-submodule with $T(M) \neq M$, then $T(M)$ is a graded prime submodule of $M$ and $(T(M)$ : $M)=0$.

Proof. By [2, Proposition 2.5 and Theorem 2.11], $T(M)$ is graded prime and $Q=(T(M): M)$ is a graded prime ideal of $R$. Now we will show that $Q=0$. Let $a=a_{g_{1}}+\ldots+a_{g_{k}} \in Q$ with $a_{g_{i}} \neq 0$. By assumption, there is a homogeneous element $m_{g}$ of $M$ with $m_{g} \notin T(M)$. Since $Q$ is graded ideal, we must have $a_{g_{i}} m_{g} \in T(M)$ for all $i$, so there are elements $0 \neq s_{h_{i}} \in h(R)$ such that $s_{h_{i}} a_{g_{i}} m_{g}=0$ for $i=1, \ldots, k$; hence for each $i, s_{h_{i}} a_{g_{i}}=0$ since $m_{g} \notin T(M)$. As $R$ is graded integral domain, we get that $a=0$, as needed.

Let $R$ be a $G$-graded ring, $M$ a graded $R$-module, $K$ a graded $R$-submodule of $M$. A graded prime $R$-submodule $N$ of $M$ is said to be a minimal graded prime of $K$ if $K \subseteq N$ and if $N^{\prime}$ is another graded prime submodle with $K \subseteq N^{\prime} \subseteq N$, then $N=N^{\prime}$. A graded $R$-module $M$ is said to be graded finitely generated if there exist $x_{g_{1}}, \ldots, x_{g_{n}} \in h(M)$ such that $M=R x_{g_{1}}+\ldots+R x_{g_{n}}$.

Lemma 2.7 Let $R$ be a $G$-graded ring, $M$ a graded $R$-module, $N$ a graded $R$-submodule of $M$. Then the following hold:

(i) If $N$ is prime, then it contains a minimal graded prime submodule.

(ii) If $M$ is a graded finitely generated $R$-module, then it has a graded maximal submodule.

Proof. (i) Since $N \subseteq N$, the set $\Delta$ of all graded prime submodules $K$ of $M$ such that $K \subseteq N$ is not empty. Of course, the relation of reverse inclusion, $\supseteq$, is a partial order on $\Delta$. Let $\Lambda$ be a non-empty subset of $\Delta$ which is totally ordered with respect to the above partial order. Then $P=\bigcap_{K \in \Lambda}$ is a proper graded submodule of $M$ since $N \neq M$. We show that $P$ is a graded prime submodule of $M$. Let $r m \in P$ where $r \in h(R)$ and $m \in h(M)-P$, so there exists $Q \in \Lambda$ such that $m \notin Q$. Let $S \in \Lambda$. Then either $Q \subseteq S$ or $S \subseteq Q$. In the first case, the facts that $r m \in Q$ implies that $r \in(Q: M) \subseteq(S: M)$, so $r \in \bigcap_{K \in \Lambda}(K: M)=(P: M)$; in the second case $r \in(S: M)$; hence $r \in(P: M)$. Thus, $P$ is an upper bound for $\Lambda$. We now use Zorn's lemma to complete the proof.

(ii) Let $x_{g_{1}}, \ldots, x_{g_{n}} \in h(M)$ be a minimal spanning set for $M$ and $N=$ $R x_{g_{2}}+\ldots+R x_{g_{n}}$ with $x_{g_{1}} \notin K$. Then the set $\Omega$ of all graded submodules $N$ 
of $M$ such that $K \subseteq N$ and $x_{g_{1}} \notin N$ is not empty since $K \in \Omega$. We therefore apply Zorn's lemma to the partially ordered set $(\Omega, \subseteq)$.

Proposition 2.8 Let $R$ be a $G$-graded ring, $M$ a graded $R$-module, $N$ a graded $R$-submodule of $M$. Then $G r(N)$ is the intersection of the minimal graded prime of $N$.

Proof. Let $K$ be a graded prime submodule of $M$ with $N \subseteq K$. Then $K / N$ is a graded prime submodule of $M / N$ by [2, Lemma 2.8]. By Lemma 2.7, there exists some graded prime submodule $S$ of $M$ such that $S / N$ is a minimal graded prime submodule $M / N$ and $S / N \subseteq K / N$; hence $N \subseteq S \subseteq K$. If $T$ is another graded prime submodule of $M$ with $N \subseteq T \subseteq S$, then in $M / N$, we must have $S / N=T / N$ so that $T=S$ in $M$. Thus, $S$ is a minimal graded prime of $N$.

Assume that $\Delta$ is the set of all grded prime submodules of $M$ containing $N$ and $\Lambda$ is the set of all minimal graded prime submodules of $N$. then the above consideration thus leads us $\operatorname{Gr}(N)=\bigcap_{K \in \Delta} K=\bigcap_{S \in \Lambda} S$.

Let $R$ be a $G$-graded ring. Graded dimension $R$ is defined by supremum of all numbers $n$ for which there exists a chain of graded prime ideals $P_{0} \subset P_{1} \subset$ $\ldots \subset P_{n}$ in $R$, and it is denoted by $\operatorname{Gdim} R$.

Theorem 2.9 If $R$ is a G-graded integral domain with $G \operatorname{dim} R=1$ and $M$ is a graded $R$-module with 0 graded prime, then either $(0: M)=0$ or every proper graded submodule of $M$ is graded prime.

Proof. Suppose $(0 ; M) \neq 0$. Since 0 is graded prime, we must have $P=$ $(0: M)$ is a graded prime ideal of $R$ by $[2$, theorem 2.11]. It follows that $P$ is a graded maximal ideal of $R$ since $\operatorname{Gdim} R=1$. If $N$ is a proper graded submodule of $M$, then $P \subseteq(N: M) \neq R$ and so $P=(N: M)$. Now the assertion follows from Proposition 2.4.

Let $R$ be a $G$-graded ring and let $M$ a graded $R$-module. Given an element $a$ of $R$, we say that $a$ divides $M$ if $a M=M$, and we say that $a$ is nilpotent on $M$ if $a^{n} M=0$ for some $n$. A non-zero graded module $M$ is graded secondary if every homogeneous element of $R$ either divides $M$ or is nilpotent on $M$, in which case $\operatorname{Gr}(\operatorname{ann} M)=P$ is a graded prime ideal of $R$, and $M$ is said to be graded $P$-secondary (see [9, Proposition 2.2]). For example, as discussed in Sharp ([9, p. 215]), if $R=k[x]$ is a polynomial ring in one variable with the natural $Z$-graded ring and $M=k[x, 1 / x]$, then $M$ is graded secondary but is 
not secondary. So the graded secondary and secondary modules are different concepts.

Theorem 2.10 If $R$ is a $G$-graded integral domain with $G \operatorname{dim} R=1$ and $M$ is a graded torsion $R$-module with 0 graded prime, then $M$ is graded secondary.

Proof. Since $M$ is a graded torsion $R$-module with 0 graded prime, we must have $(0: M)=P \neq 0$ is a graded prime ideal. Let $a \in h(R)$. If $a \in P$, then $a$ is nilpotent on $M$. Now we show that $a$ divides $M$. Suppose not. Then by Theorem $2.9,(a M: M)=P$, so $a \in P$ which is a contradiction.

Let $M$ be a graded module over a $G$-graded ring $R$. Note that some graded modules $M$ have no graded prime submodules and we call such modules $M$ $G$-primeless. There are many examples as we show next. A graded $R$-module $M$ is called $G$-divisible (resp. graded torsion module) if $a M=M$ for all $0 \neq a \in h(R)($ resp. $T(M)=M)$.

Theorem 2.11 Let $R$ be a $G$-graded integral domain. Then every graded torsion $G$-divisible $R$-module is $G$-primeless.

Proof. Assume that $M$ is a $G$-divisible graded torsion $R$-module. Suppose that $M$ is not $G$-primeless; we show that $M=N$. Let $N$ be a graded prime submodule of $M$ with $(N: M)=P$. First, we show that $P=0$. Otherwise, there is an element $r=\sum_{i=1}^{n} r_{g_{i}} \in P$ with $r_{g_{i}} \neq 0$, so for each $i$, we must have $r_{g_{i}} \in P$ since $P$ is graded ideal; hence by assumption, we get $M=r_{g_{i}} M \subseteq N$ which is a contradiction. Therefore, by [2, Theorem 2.11], $M / N$ is a graded torsion-free $R$-module. Next, we show that $M / N$ graded torsion-free gives $M \subseteq N$. Suppose that $M \nsubseteq N$. Then there is a homogeneous element $m_{g}$ of $M$ such that $m_{g} \notin N$, so $r\left(m_{g}+N\right) \neq 0$ for all non-zero element $r$ of $h(R)$; hence $r m_{g} \notin N$ for all $0 \neq r \in h(R)$. On the other hand, as $M$ is graded torsion, there is a non-zero homogeneous element $s$ of $R$ such that $s m_{g}=0 \in N$. Thus, $M=N$ which is a contradiction.

Theorem 2.12 Let $M$ be a G-primeless module over a G-graded integral domain $R$. Then $M$ is graded torsion, $P M=M$ for every graded maximal ideal $P$ of $R$ and $M$ is not a graded finitely generated $R$-module.

Proof. If $T(M) \neq M$, then $T(M)$ is a graded prime submodule of $M$ by Proposition 2.6. Thus $M$ is graded torsion. By Proposition 2.4, we must have $P M=M$ for every graded maximal ideal $P$ of $R$. Finally, $M$ is not graded 
finitely generated by Lemma 2.7 (since every graded maximal submodule is graded prime by [4, Proposition 2.6]).

Let $R$ be a $G$-graded ring, $M$ a graded $R$-module, $N$ a graded $R$-submodule of $M$. We say that $N$ has a reduced graded primary decomposition if there are finitely many graded primary submodules $N_{i}$ such that $N=\bigcap_{i=1}^{n} N_{i}$ with $\bigcap_{i \neq j} N_{i} \nsubseteq N_{j}$ for each $i$ and the graded prime ideals $\operatorname{Gr}\left(N_{i}: M\right)$ are distinct. In this case, we say that $\operatorname{Gr}\left(N_{i}\right)$ is an isolated prime submodule of $N$ if it is minimal the set $\left\{G r\left(N_{1}\right), \ldots, G r\left(N_{i}\right)\right\}$.

Theorem 2.13 Let $R$ be a $G$-graded ring, $M$ a graded $R$-module, $N$ a graded $R$-submodule of $M$. If $N$ has a reduced graded primary decomposition $N=$ $\bigcap_{i=1}^{n} N_{i}$ such that all the graded prime ideals associated with $N$ are isolated, then $(N: M)=\sum_{i=1}^{n}\left(N_{i}: M\right)$ is a reduced graded primary decomposition of the graded ideal $(N: M)$ in $R$.

Proof. Suppose not. As the graded ideals $\operatorname{Gr}\left(N_{i}: M\right)$ are distinct, there exists integer $i$ such that $\bigcap_{i \neq j}\left(N_{j}: M\right) \subseteq\left(N_{i}: M\right)$, so by [8, Proposition 1.2], $\bigcap_{i \neq j} \operatorname{Gr}\left(N_{j}: M\right) \subseteq G r\left(N_{i}: M\right)$; hence $\operatorname{Gr}\left(N_{j}: M\right) \subseteq\left(N_{i}: M\right)$ for some $j \neq i$ by [8, Lemma 1.8 and proposition 1.4]. However, this final inclusion contradicts the assumption that $\operatorname{Gr}\left(N_{i}: M\right)$ is an isolated graded prime ideal of $N$.

Proposition 2.14 Let $R$ be a G-graded ring, $M$ a graded $R$-module, $N$ a graded $R$-submodule of $M$. If $N$ has a reduced graded primary decomposition $N=\bigcap_{i=1}^{n} N_{i}$ such that all the graded prime ideals associated with $N$ are isolated, then the following hold:

(i) $N$ is grded primary if and only if $(N: M)$ is graded primary.

(ii) $N$ is grded prime if and only if $(N: M)$ is graded prime.

Proof. (i) If $N$ is graded primary, then $(N: M)$ is graded primary by [4, Proposition 2.7]. conversely, assume that $(N: M)$ is a graded primary ideal of $R$. By Theorem 2.13, $(N: M)=\sum_{i=1}^{n}\left(N_{i}: M\right)$ is a reduced graded primary decomposition of $(N: M)$, so we must have $n=1$ and so $N=N_{1}$ is primary.

(ii) The necessity follows from [4, Proposition 2.7]. If $(N: M)$ is graded prime, then $N=N_{1}$ is a primary submodule, so $(N: M)=\operatorname{Gr}(N: M)$ by [8, proposition 1.2]. Suppose $a x \in N$ with $a \in h(R)$ and $x \in h(M)-N$. Then $N$ graded primary gives $a \in G r(N: M)=(N: M)$; hence $N$ is a prime submodule of $M$. 
Lemma 2.15 Let $R$ be a G-graded ring. Then $R$ is a graded field if and only if every proper graded ideal is graded prime.

Proof. If $R$ is a graded field, then the result follows from Lemma 2.3. Conversely, Let $c$ be a non-zero element of $h(R)$. By assumption, $R c^{2}$ is a grded prime ideal of $R$, so $c^{2} \in R c^{2}$ implies $c \in R c^{2}$; hence there exists $a=a_{g_{1}}+\ldots+a_{g_{k}} \in R$ with $a_{g_{i}} \neq 0$ such that $c=c^{2} a$. It follows that $c=c^{2} a_{g_{j}}$ for some $j$. As the graded ideal $\{0\}$ is prime, we must have $R$ is a $G$-graded integral domain, so we can c ancel $c$ to obtain $c a_{g_{j}}=1$, showing that $R$ is $G$-graded field.

Theorem 2.16 Let $R$ be a $G$-graded ring and $0 \neq M$ a graded $R$-module, then $R$ is a graded field if and only if every proper graded submodule of $M$ is a graded prime submodule of $M$ and $T(M) \neq M$.

Proof. Let $R$ be a graded field. First, suppose that $T(M)=M$ and $m=$ $m_{g_{1}}+\ldots+m_{g_{n}} \in M$ with $m_{g_{i}} \neq 0$. There are non-zero homogeneous elements $r_{g_{i}}$ of $R$ such that $r_{g_{i}} m_{g_{i}}=0$ for $i=1, \ldots, n$, so for each $i, m_{g_{i}}=0$ since $R$ is graded field; hence $M=0$ which is a contradiction. Thus $T(M) \neq M$. Next, assume that $N$ is a proper graded submodule of $M$ and let $a x \in N$ where $a \in h(R)$ and $x \in h(M)$. We can assume that $a \neq 0$. Then $x \in N$ since $R$ is graded field. Conversely, let $c \in M-T(M)$, so there is a homogeneous component $c_{g} \neq 0$ of $c$ such that $c_{g} \notin T(M)$; hence $\operatorname{Ann}\left(c_{g}\right)=0$. In view of the assumption, it is easy to see that every proper graded submodule of the graded $R$-module $R c_{g}$ is a graded prime submodule of $R c_{g}$ and $R c_{g} \cong R$ as $R$-modules. Therefore, every proper graded ideal of $R$ is a graded prime ideal; hence $R$ is graded field by Lemma 2.15 .

Corollary 2.17 If $R$ is a $G$-graded integral domain with $G \operatorname{dim} R=1$ and $M$ is a graded $R$-module with $(0: M) \neq 0$ and 0 graded prime, then $R /(0: M)$ is graded field.

Proof. This follows from Theorem 2.9 and Lemma 2.3.

A $G$-graded ring $R$ is said to be $G$-graded quasi-local if it has a unique graded maximal ideal.

Lemma 2.18 If $R$ is a G-graded ring in which every proper graded ideal is graded primary, then $R$ is a graded quasi-local. 
Proof. Let $P$ and $I$ be graded $R$-ideals with $P$ graded prime. We show that either $P \subseteq I$ or $I \subseteq P$. Assume that $I \nsubseteq P$ and choose $r \in I-P$. Then there exists a homogeneous component $r_{g} \neq 0$ of $r$ such that $r_{g} \notin P$. Let $a=a_{h_{1}}+\ldots, a_{h_{k}} \in P$ with $a_{h_{i}} \neq 0$. Then for each $i, r_{g} a_{h_{i}} \in I P$ and $I P$ is graded primary ideal, so we must have either $a_{h_{i}} \in I P$ or $r_{g}^{m} \in I P$ for some $m$. But if $r_{g}^{m} \in I P \subseteq P$, then $r_{g} \in P$ which is a contradiction. Thus we must have for each $i, a_{h_{i}} \in I P$; hence $a \in I P \subseteq I$. Thus, $P \subseteq I$. Let $M$ be any graded maiximal ideal (so it is a graded prime ideal) of $R$. Then it is comparable to any proper graded ideal. Now if $Q$ is any maximal ideal, then $M$ and $Q$ are comparable, so $M=Q$, as needed.

Theorem 2.19 Let $R$ be a G-graded ring and $0 \neq M$ a graded $R$-module. If every proper graded submodule of $M$ is a graded primary submodule of $M$ and $T(M) \neq M$, then $R$ is graded quasi-local.

Proof. As $T(M) \neq M$, there is a homogeneous element $0 \neq b_{g} \in h(M)$ such that $b_{g} \notin T(M)$ and $A n n\left(b_{g}\right)=0$; hence $R b_{g} \cong R$ as $R$-modules. Clearly, every proper graded submodule of the graded $R$-module $R b_{g} \cong R$ is a graded primary submodule; hence $R$ is graded quasi-local by lemma 2.18.

\section{References}

[1] S. Ebrahimi Atani: On graded weakly prime submodules, International Mathematical Forum, 1- 4 (2006), 61-66.

[2] S. Ebrahimi Atani: On graded prime submodules, Chiang Mai J. Sci., 33(1) (2006). 3-7.

[3] S. Ebrahimi Atani: On graded weakly prime ideals, Turkish Journal of Mathematics, 30 (2006), 1-8.

[4] S. Ebrahimi Atani and F. Farzalipour: On graded secondary modules, submmited.

[5] R. L. McCasland, M. E. Moor and P. F. Smith, On the spectrum of a module over a commutative ring, Comm. Algebra 25(1) (1997), 79-103.

[6] M. E. Moor and S. J. Smith, Prime and radical submodules of modules over commutative rings, Comm. Algebra 30(10) (2002), 5037-5064. 
[7] C. Nastasescu and F. Van Oystaeyen: Graded Ring Theory, Mathematical Library 28, North Holand, Amsterdam, (1982).

[8] M. Refai and K. Al-Zoubi: On Graded Primary Ideals, Turkish Journal of Mathematics, 28 (2004), 217-229.

[9] R. Y. Sharp: Asmptotic of certain sets of attached prime ideals, J. London Math. Soc., 34 (1986), 212-218.

Received: May 4, 2006 